\title{
Verbesserung der Klimabilanzen landwirtschaftlicher Betriebe und der Beitrag der Digitalisierung
}

\author{
Clemens Fuchs • Paul Gütschow • Rainer Langosch • Katharina Skau • \\ Laura Thierbächer
}

Eingegangen: 27. Oktober 2020 / Angenommen: 3. Dezember 2020 / Online publiziert: 15. Dezember 2020

(C) Der/die Autor(en) 2020

Zusammenfassung Eine nachhaltige Landbewirtschaftung kann ihren Beitrag zum Klimaschutz leisten. Die internationalen Vereinbarungen zum Klimaschutz finden ihren Niederschlag in der EU-Politik, z. B. der Farm-to-Fork-Strategie. Die Digitalisierung kann eine wichtige Unterstützung für die notwendige Anpassung von Produktionsprozessen sein. Auch die Landwirte selbst sehen im zunehmenden ITEinsatz Möglichkeiten zur Verbesserung der Ressourceneffizienz. An zwei Praxisbeispielen, einem Ackerbaubetrieb und einen ökologischen Milchviehbetrieb wird modellhaft beschrieben, wie die Reduzierung des $\mathrm{CO}_{2}$-Fußabdruck mit Hilfe der Digitalisierung erreicht werden kann. Die Notwendigkeit zur Optimierung bei Netzabdeckung und zur Verbesserung der Kompatibilität von Systemen, die Problematik von langfristigen Investitionszyklen, welche mit der schnelllebigen Digitalisierungsumwelt kollidieren sowie z.B. bislang ungeklärte Fragen der Datenhoheit werden diskutiert. Abschließend wird herausgestellt, dass die Vorteile der IT nur dann voll zur Wirkung kommen, wenn die Nutzer qualifiziert werden und Beratung erhalten.

Schlüsselwörter Digitalisierung $\cdot \mathrm{CO}_{2}$-Fußabdruck $\cdot$ Ackerbau $\cdot$ Milchviehhaltung

C. Fuchs $(\varangle) \cdot$ P. Gütschow $\cdot$ R. Langosch $\cdot$ K. Skau $\cdot$ L. Thierbächer

Fachbereich Agrarwirtschaft und Lebensmittelwissenschaften, Hochschule Neubrandenburg,

University of Applied Sciences, Neubrandenburg, Deutschland

E-Mail: cfuchs@hs-nb.de 


\title{
Action plan on food price volatility and agriculture
}

\begin{abstract}
Sustainable land management can make its contribution to climate protection. The international agreements on climate protection are reflected in EU politics, e.g. the farm-to-fork strategy. Digitalization can be an important support for the necessary adaptation of production processes. The farmers themselves also see opportunities to improve resource efficiency in the increasing use of IT. Two practical examples, an arable farm and an organic dairy farm, are used to describe how the reduction of the $\mathrm{CO}_{2}$ footprint can be achieved with the help of digitalization. The need to optimize network coverage and improve the compatibility of systems, the problem of long-term investment cycles that collide with the fast-moving digitization environment and e.g. So far unresolved questions of data sovereignty are finally discussed. Finally, it is pointed out that the advantages of IT can only be fully effective if the users are qualified and receive advice.
\end{abstract}

Keywords Digitalization · Carbon footprint $\cdot$ Arable farming $\cdot$ Dairy farming

\section{Einleitung}

„Wir erinnern an die dreifache Herausforderung für die Landwirtschaft: Sicherung der Ernährung bei gleichzeitiger Anpassung an den Klimawandel und Reduzierung des Beitrags zur Emission von Treibhausgasen." so heißt es in der Abschlusserklärung der G20-Agrarminister 2011 in Paris (Meeting of G20 Agriculture Ministers 2011). Um die Gefahren des Klimawandels zu reduzieren fordert das Pariser Klimaabkommen eine Begrenzung der Erderwärmung auf $1,5^{\circ} \mathrm{C}$. Dazu ist es notwendig die globalen Emissionen bis 2030 um $45 \%$ gegenüber $2010 \mathrm{zu}$ reduzieren und bis 2050 Netto-Nullemissionen zu realisieren.

Die Welt erscheint in der öffentlichen Wahrnehmung weit davon entfernt dieses Ziel zu erreichen. Das lässt sich zumindest aus den seit Sommer 2018 stattfindenden Protesten Jugendlicher und junger Erwachsener heraushören, die sich im Rahmen der Fridays for Future-Bewegung für eine schnelle und effiziente Umsetzung von Klimaschutzmaßnahmen einsetzen. Aus der Perspektive der Landwirtschaft ist indessen zu bemerken, dass eine globale Entwicklung im Sinne ökologischer, ökonomischer und sozialer Nachhaltigkeit mit einem hohen Maß an Komplexität der Zielstellung einhergeht. So zeigen die 17 UN-Nachhaltigkeitsziele der Agenda 2030 (SDG 17) ${ }^{1}$ die hohen Anforderungen an kohärente und konsistente Zielsysteme und Strategien. Klimaschutz ist ein wichtiges, nicht jedoch das einzige Ziel.

Eingriffe in natürliche Kreisläufe führen zu Veränderungen von Stoffströmen und Emissionen. Der Club of Rome hat kalkuliert, dass in einer Welt von Jägern und

\footnotetext{
1 Die 17 Ziele für eine nachhaltige Entwicklung beruhen auf der Idee globaler Partnerschaften von entwickelten und sich entwickelnden Ländern zur Beendigung von Armut, Verbesserung von Gesundheit und Bildung, Veringerung von Ungleichheit, Förderung des Wirtschaftswachstums, Bekämpfung des Klimawandels, Erhalt der Ozeane und Wälder. Die Agenda 2030 wurde 2015 von allen Mitgliedstaaten der Vereinten Nationen verabschiedet (UN 2015).
} 
Sammlern 20Mio. Menschen Nahrung fänden (Radermacher 2015). Derzeit sind allerdings weltweit knapp 7,9 Mrd. Menschen darauf angewiesen, dass ihre Zukunft auch aus Perspektive des Weltklimas, aber darüber hinaus eben auch aus der Perspektive ausreichender Nahrung, ausreichenden Trinkwassers, angemessener Entwicklung und der anderen Ziele der SDG 17 politisches Handeln erfordert. Etwaige Forderungen nach einer ,Null-Emissionslandwirtschaft“ würden also an den realen Möglichkeiten einer global wachsenden Weltbevölkerung vorbeigehen. Realistischer - und insofern auch schlüssiger - wäre eine deutliche Reduktion der Klimawirkungen der Landwirtschaft. Vor diesem Hintergrund sind die Anforderungen und auch die Untersuchung wichtiger Handlungsoptionen der Landwirtschaft im Interesse des Klimaschutzes zu würdigen.

Das von der EU unterzeichnete Pariser Abkommen ist der Grundstein für weitere national festgelegte Leitlinien im Umgang mit dem Klimawandel. Das Klimagesetz der EU sieht vor die EU bis 2050 klimaneutral zu stellen und die Nettotreibhausgasemissionen bis 2030, um mindestens 55\% im Vergleich zu 1990 zu reduzieren. Die hauptsächlich von der Landwirtschaft emittierten Treibhausgase (THG) sind Kohlenstoffdioxid $\left(\mathrm{CO}_{2}\right)$, Methan $\left(\mathrm{CH}_{4}\right)$ und Lachgas $\left(\mathrm{N}_{2} \mathrm{O}\right)^{2}$. Aus Untersuchungen des Umweltbundesamtes (2020) geht hervor, dass rund $62 \%$ der Methan- und Lachgasemissionen in Deutschland auf die Landwirtschaft zurück zu führen sind. Dabei werden die Methanausstöße hauptsächlich der Viehhaltung zugeschrieben, während die direkten und indirekten Lachgasemissionen zum größten Teil aus der Düngung der Acker- und Grünlandflächen sowie den Auswaschungen von Stickstoffverbindungen aus Oberflächenabfluss und Auswaschung von gedüngten Flächen stammen. Auf europäischer Ebene sind 10,3\% der Treibhausgasemissionen (THG-Emissionen) der Landwirtschaft zuzurechnen, wobei $70 \%$ davon aus der Tierhaltung stammen (EUKommission 2020).

Zur Verminderung der Treibhausgasemissionen widmet das Konzept des Greendeals ${ }^{3}$ der EU dem Agrarsektor besondere Aufmerksamkeit. Die „Farm-to-ForkStrategie" soll gewährleisten, dass Landwirtschaft, Fischerei und Aquakultur sowie die Lebensmittelwertschöpfungskette hinreichend zur Reduktion der THG-Emissionen beitragen. Dazu sollen Bewirtschaftungsmethoden, mit denen Kohlendioxid aus der Atmosphäre entfernt wird, durch Zahlungen im Rahmen der gemeinsamen EU-Agrarpolitik oder im Wege anderer öffentlicher oder privater Initiativen $\left(\mathrm{CO}_{2-}\right.$ Zertifikate-Handel) entlohnt werden. Im Rahmen der aktuellen europäischen Verhandlungen zu einer neuen gemeinsamen Agrarpolitik bis 2027 ist vorgesehen, dass $40 \%$ des Gesamtbudgets des Agrarhaushalts zum Klimaschutz beitragen werden (EU-Kommission 2020).

Dass Nachhaltigkeit und Klimaschutz einen großen Stellenwert in der Landwirtschaft besitzen unterstreicht eine aktuelle mit 500 Landwirten durchgeführte Studie

\footnotetext{
2 Deren (relatives) Treibhauspotential (global warming potential, GWP) wird über einen 100 Jahreszeitraum mit dem Faktor 1 für Kohlenstoffdioxid $\left(\mathrm{CO}_{2}\right), 34$ für Methan $\left(\mathrm{CH}_{4}\right)$ und 289 für Lachgas $\left(\mathrm{N}_{2} \mathrm{O}\right)$ in $\mathrm{CO}_{2}$ - Äquivalente $\left(\mathrm{CO}_{2}\right.$ eq) umgerechnet (Myhre et al. 2013).

${ }^{3}$ Der Green Deal ist ein wesentlicher Bestandteil der Strategie der EU-Kommission zur Umsetzung der Agenda 2030 der Vereinten Nationen (UN) im Rahmen der 17 Ziele für eine nachhaltige Entwicklung (SDG's). Die 17 SDG's knüpfen an die Milleniumsentwicklungsziele (MDG's) der UN von 2000 bis 2015 an.
} 
des Bundesverbandes Informationswirtschaft, Telekommunikation und neue Medien e. V. (Bitkom). $62 \%$ der Befragungsteilnehmenden gehen davon aus, dass die Landwirtschaft insgesamt eine große Bedeutung im Kampf gegen den Klimawandel hat. Jeder zweite Befragte wäre dazu bereit die Produktion aufgrund des Klimawandels anzupassen. Große Erwartungen richten sich an den Beitrag der IT in diesem Prozess. So zeigen sich $93 \%$ der befragten Landwirte davon überzeugt, dass Verbesserungen in der Ressourceneffizienz durch den Einsatz digitaler Technologien zur Einsparung von Dünger und Pflanzenschutzmitteln möglich sind. Wobei insgesamt $81 \%$ der Befragungsteilnehmenden davon ausgehen, dass die Produktion mithilfe der Digitalisierung umweltschonender wird (Bitkom Research 2020).

Der verantwortungsvolle Umgang mit den natürlichen Ressourcen (Ökologie) bei gleichzeitiger Sicherung der Ernährung (Soziales) und Wirtschaftlichkeit (Ökonomie) ist Grundvoraussetzung für eine nachhaltige Produktionsweise. Die Digitalisierung der Landwirtschaft könnte im Interessenausgleich dieser drei Dimensionen eine zunehmend wichtigere Bedeutung einnehmen.

Dieser Beitrag beschäftigt sich mit den möglichen Ansatzstellen der Digitalisierung zur Verbesserung der Klimabilanz in der Landwirtschaft. Dazu wird der Stand der Digitalisierung in der Landwirtschaft beschrieben (Kap. 2) und am Beispiel der Potenziale zur Reduzierung des $\mathrm{CO}_{2}$-Fußabdruckes der Produktionsrichtungen Ackerbau (Kap. 3) und Milchviehhaltung (Kap. 4) eingeordnet. Abschließend werden Umsetzungsvoraussetzungen und Erfolgsbedingungen für den Einsatz digitaler Anwendungen in einer marktwirtschaftlich-unternehmerisch geprägten Landwirtschaft beschrieben (Kap. 5) und zusammenfassend beurteilt (Abschn. 5.1).

\section{Aktueller Stand der Digitalisierung in der Landwirtschaft}

Bereits heute setzten die meisten landwirtschaftlichen Betriebe digitalen Technologien ein (Abb. 1). Die Landwirte selbst haben relativ hohe Erwartungen an die digitalen Technologien und sehen große Potenziale für Tierwohl, Nachhaltigkeit und Umwelt. Sie erwarten, dass sich damit Dünger, Pflanzenschutzmittel und andere Ressourcen einsparen lassen und so eine umweltschonende Produktion möglich

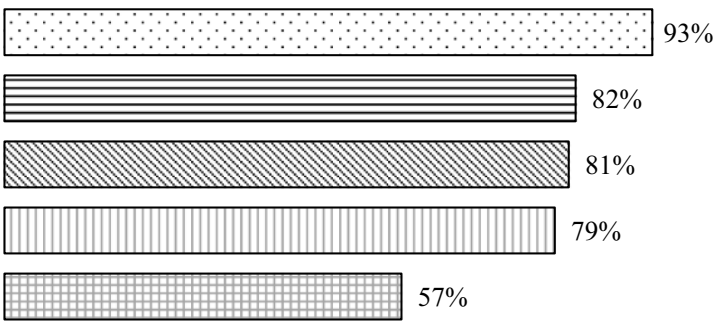

$0 \%$
$20 \%$

$40 \%$

\section{Digitale Technologien:}

$\square$ helfen Dünger, Pflanzenschutzmittel
und andere Ressourcen einzusparen,
$\square$ landwirtschaftliche Betriebe setzen
sie ein,
घ ermöglichen eine umweltschonende
Produktion,
$\square$ bieten körperliche Entlastung und
$\square$ es lassen sich Beruf und Privatleben
besser vereinen.

Abb. 1 Beurteilung der digitalen Technologien durch Landwirte; Befragung von 500 Landwirten im Februar/März 2020; Mehrfachnennungen möglich, (Quelle: Bitkom Research 2020) 
82\% der landwirtschaftlichen Betriebe setzen digitale Technologien ein; häufigste Einsatzgebiete:

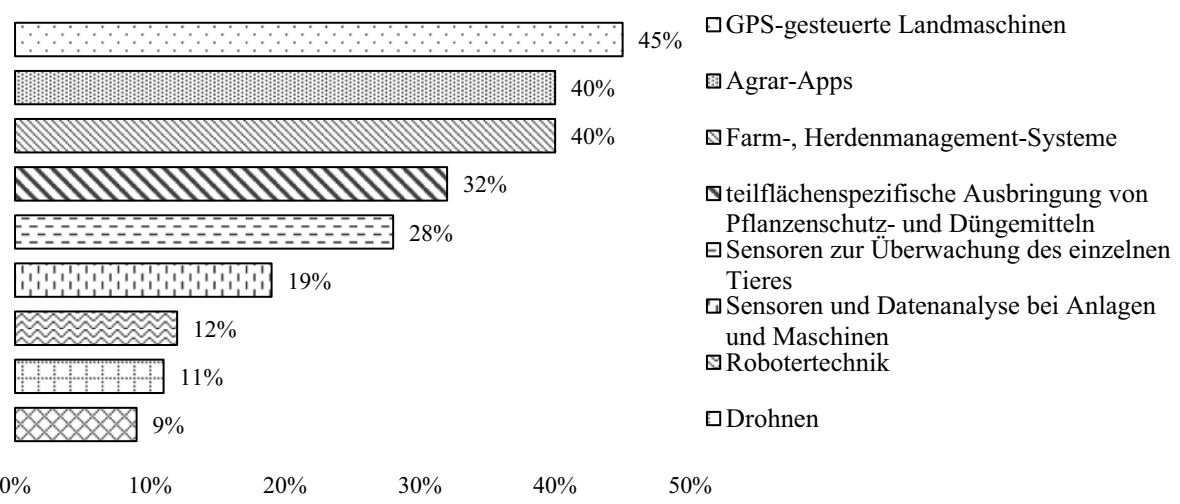

Abb. 2 Häufigste Einsatzgebiete digitaler Technologien in der Landwirtschaft; Befragung von 500 Landwirten im Februar/März 2020; Mehrfachnennungen möglich, (Quelle: Bitkom Research 2020)

wird. Von den neuen Technologien werden körperliche Entlastung erwartet und dass sich Beruf und Privatleben besser vereinen lassen werden.

Bereits $82 \%$ der landwirtschaftlichen Betriebe setzen digitale Technologien ein. Hightech-Landmaschinen, Agrar-Apps, Robotik oder Drohnen sind heute ein fester Bestandteil der Landwirtschaft (Abb. 2). Die gezielte Steuerung und Kontrolle von Teilflächen bei der Ausbringung von Pflanzenschutz- und Düngemitteln sowie die Überwachung des einzelnen Tieres und von Anlagen und Maschinen gelingt mit dem Einsatz von digitalen Sensoren und der elektronischen Weiterverarbeitung der erhobenen Daten.

Etwa drei Viertel $(73 \%)$ der Betriebe sehen in der Digitalisierung grundsätzlich eine Chance. $64 \%$ betonen, so könnten langfristig Kosten gesenkt werden. Gleichwohl ist die Digitalisierung für mehr als jeden zweiten Landwirt (58\%) eine große Herausforderung. 40\% verzeichnen zudem einen Mangel an Mitarbeitern mit digitalem Know-how und $17 \%$ betrachten die Digitalisierung gar als Risiko.

$88 \%$ der befragten Landwirte erwarten, dass die Digitalisierung auch für mehr Transparenz gegenüber dem Verbraucher sorgt. Deutlich weniger Landwirte nutzen jedoch digitale Techniken, um aktiv Verbraucher anzusprechen. Jeder Vierte Landwirt (24\%) ist in sozialen Netzwerken aktiv, jeder Fünfte (19\%) hat eine eigene Website. $16 \%$ bieten eine Online-Rückverfolgbarkeit „vom Hof bis zum Teller“ an - und jeder Zehnte (10\%) hat Webcams im Stall oder auf dem Feld installiert. $9 \%$ vermarkten ihre Produkte über eigene digitale Hofläden oder Plattformen (Bitkom Research 2020).

Technischer Fortschritt in Form digitaler Anwendungen könnte dazu beitragen bestehende Effizienzlücken in bestimmten Bereichen der Landwirtschaft identifizieren und schließen zu können. So deutet eine Untersuchung zur Produktivität von 141 norddeutschen Marktfruchtbetrieben unter Berücksichtigung von THG-Emissionen darauf hin, dass die Reduzierung von produktspezifischen THG-Emissionen von bis zu 8,6\% ohne zusätzliche Kosten möglich sein könnte (Wettemann 2017). 
Die langfristige Reduzierung von THG-Emissionen wäre ein wichtiger Beitrag der Landwirtschaft zum Klimaschutz. Weltweit trägt die Landwirtschaft zu ca. $10 \%$ bis $12 \%$ zu den anthropogenen THG-Emissionen bei (Smith et al. 2014). Um vorhandene Potentiale einer angepassten Produktionsweise verdeutlichen zu können, werden nachfolgend für den Ackerbau und für die Milchproduktion Modellrechnungen für zwei landwirtschaftliche Betriebe in Mecklenburg-Vorpommern dargestellt. Dabei wird herausgestellt, inwieweit die Digitalisierung einen Beitrag zur Emissionsreduktion leisten könnte.

\section{$3 \mathrm{CO}_{2}$-Fußabdruck im Ackerbau und Möglichkeiten der Reduzierung}

Für das Produktionsverfahren Weizenbau stellt das Julius-Kühn-Institut (JKI) - Bundesinstitut für Kulturpflanzen in einem langjährigen Feldversuch in Bezug auf den Einfluss von Pflanzenschutzstrategie und Bodenbearbeitung auf den $\mathrm{CO}_{2}$-Fußandruck von Weizen fest, dass im Durchschnitt über sämtliche Versuchsvarianten und Jahre die THG-Emissionen bei $3002 \mathrm{~kg} \mathrm{CO}_{2}$ eq ha ${ }^{-1}$ und der $\mathrm{CO}_{2}$-Fußabdruck (CFP) bei $0,53 \mathrm{~kg} \mathrm{CO}_{2} \mathrm{eq} \mathrm{kg}^{-1}$ lagen (Feike et al. 2020). Untersucht wurde unter anderem, ob der Verzicht auf den Pflug (wendende Bodenbearbeitung) und die dadurch möglichen Einsparungen beim Diesel ein nachhaltiges Wirtschaften ermöglicht. Im Vergleich zu den nicht-wendenden Varianten (Einsatz des Grubbers anstatt des Pfluges) war jedoch kein signifikanter Unterschied bei den THG-Emissionen feststellbar. Dagegen führt bezüglich des Einsatzes von Pflanzenschutzmitteln die ertragssichernde Wirkung der Fungizide zu einem geringen $\mathrm{CO}_{2}$-Fußabdruck in der Weizenproduktion.

Der $\mathrm{CO}_{2}$-Fußabdruck einer Kultur wie beispielsweise Winterweizen oder die sich auf die $\mathrm{CO}_{2}$-Bilanz eines Betriebes auswirkende Veränderung eines Betriebsmittels wie Diesel werden und wurden in verschiedenen Projekten untersucht. Das Projekt „EKoTech - Effiziente Kraftstoffnutzung der Agrartechnik“ fand heraus, dass sich der Dieselbedarf für die Erzeugung einer Tonne Winterweizen von 1990 bis heute um $30 \%$ verringert hat. Der kraftstoffbezogene $\mathrm{CO}_{2}$-Fußabdruck hat sich somit erheblich verbessert und zeigt, dass die moderne Landtechnik wesentlich dazu beiträgt, Klimapotentiale zu heben (Scherer 2020; siehe auch: EKoTech - Effiziente Kraftstoffnutzung der Agrartechnik).

Feike et al. (2020) nutzen in ihren Berechnungen eine partielle Lebenszyklusanalyse. Dabei umfassen die Systemgrenzen den vorgelagerten Bereich - Maschinen, Saatgut, Düngemittel und Pflanzenschutzmittel - die Emissionen auf dem Feld und den nachgelagerten Bereich, zu dem der Korntransport zum Hof zählt. Die Autoren untersuchen die drei Faktoren Pflanzenschutzstrategie (Gute fachliche Praxis vs. Integrierter Pflanzenschutz), Fungizidvariante (mit und ohne) und Bodenbearbeitungsvariante (wendend und nicht wendend) und deren Einfluss auf die THGEmissionen über 12 Jahre in Feldversuchen. Dabei wird deutlich, dass die wendende Bodenbearbeitung nicht per se schlechter in Bezug auf Klimagase ist als die nicht wendende. Außerdem zeigt sich, dass ein Verzicht auf Fungizide eindeutig negative Auswirkungen auf die Ertragssicherung und damit auf den $\mathrm{CO}_{2}$-Fußabdruck von Weizen hat. 
Brankatschk und Finkbeiner (2017) entwickeln die einjährige Lebenszyklusanalyse weiter und beziehen Fruchtfolgeeffekte und Ernterückstände über längere Zeiträume in ihre Berechnungen mit ein. Betrachtet werden die Endprodukte Weizenbrot, Milch und Biodiesel. Die Berechnungen zeigen, dass bei allen drei Produkten der $\mathrm{CO}_{2}$-Fußabdruck deutlich verbessert wird, wenn die Fruchtfolgeeffekte berücksichtigt werden. Bei einjähriger Betrachtung zum Beispiel wird Stickstoffdünger belastend in die Berechnungen aufgenommen, bei mehrjähriger Betrachtung hingegen wird beispielsweise der mögliche Stickstofftransfer zwischen den einzelnen Kulturen entgegengesetzt. Auch Bodenfruchtbarkeit und Effekte auf Bodenorganismen, die die $\mathrm{CO}_{2}$-Ergebnisse beeinflussen, können in diese Berechnungen aufgenommen werden. Wird Stroh als Ko-Produkt gesehen und vom Feld abgefahren anstatt als „Abfallprodukt“ nicht berücksichtigt zu werden, verändert sich der $\mathrm{CO}_{2}$-Fußabdruck von beispielsweise Biodiesel innerhalb einer Fruchtfolgerotation zum Negativen.

Neben den verschiedenen Varianten der Bodenbearbeitung und des Pflanzenschutzes gibt es noch weitere ackerbauliche Komponenten, z. B. die Rotation der Kulturen, die in eine nachhaltige Produktionsstrategie einfließen sollten. Modellhaft wird dies für einen Betrieb in Mecklenburg-Vorpommern betrachtet. Mit rund 800 ha Betriebsfläche handelt es sich um einen überdurchschnittlich großen Betrieb, da die landwirtschaftlichen Betriebe in Deutschland im Mittel rund 60 ha und in Mecklenburg-Vorpommern im Mittel 274 ha bewirtschaften.

Der Beispielbetrieb liegt im Landkreis Mecklenburgische Seenplatte in Mecklenburg-Vorpommern. Die Bonität der Betriebsflächen liegt mit durchschnittlich bei 42 Bodenpunkten im Mittelmaß, da die Skala bis 104 Bodenpunkte reicht, und ist bestimmt durch die Bodenarten lehmiger Sand (IS) bis sandiger Lehm (sL). Auf den rund 800 ha Ackerfläche wird eine fünfgliedrige Fruchtfolge angewandt, in der Winterweizen die stärkste Anbaufrucht darstellt, gefolgt von Winterraps, Wintergerste und Winterroggen. Des Weiteren werden auf einem Teil der Flächen die Sommerkulturen Erbse und Mais angebaut. Im Erntejahr 2019 werden in den Berechnungen für die Darstellung des Status quo Winterweizen, Wintergerste, Winterraps und Futtererbsen dargestellt.

Die Bewirtschaftung erfolgt nach den „Grundsätzen der guten fachlichen Praxis“ in Bezug auf Bodenbearbeitung, Düngung nach Entzug und Pflanzenschutz, der sich an Schadschwellen orientiert (Bundesministerium für Ernährung und Landwirtschaft 2019). Die Aufwendungen für Pflanzenschutzmittel waren im Frühjahr 2019 im Vergleich zu anderen Jahren relativ gering, was auf die Trockenheit in jenem Jahr zurück zu führen ist. Für die Berechnungen werden sowohl beim Düngereinsatz als auch beim Einsatz von Pflanzenschutzmitteln Mittelwerte für die jeweilige Kultur gebildet, die aus der Ackerschlagkartei des Betriebes stammen. Für das Erntejahr 2019 wurden folgende Anbauumfänge verzeichnet (Tab. 1).

In Bezug auf den aktuellen Verbrauch fossiler Energien und einem möglichen späteren Ersatz durch Erneuerbare Energien sei an dieser Stelle auch ein Hinweis auf den Maschineneinsatz gestattet. Der Betrieb ist mit vier Traktoren, einem Mähdrescher und einem Teleskoplader voll eigenmechanisiert und lagert die Ernte nahezu vollständig ein. Die Trocknung der Erntefrüchte findet - bei Bedarf - selbstständig statt, wobei die Trocknungsanlage mit Heizöl betrieben wird. 
Tab. 1 Fruchtfolgeanteile in der Ausgangssituation, Beispielbetrieb im Erntejahr 2019. (Quelle: Betriebsdaten)

\begin{tabular}{lllllll}
\hline Vorfrucht 2018 & $\begin{array}{l}\text { Winter- } \\
\text { raps }\end{array}$ & $\begin{array}{l}\text { Winter- } \\
\text { weizen }\end{array}$ & $\begin{array}{l}\text { Winter- } \\
\text { weizen }\end{array}$ & $\begin{array}{l}\text { Winter- } \\
\text { weizen }\end{array}$ & $\begin{array}{l}\text { Winter- } \\
\text { gerste }\end{array}$ & $\begin{array}{l}\text { Winter- } \\
\text { weizen }\end{array}$ \\
$\begin{array}{l}\text { Anbau im Ernte- } \\
\text { jahr 2019 }\end{array}$ & $\begin{array}{l}\text { Winter- } \\
\text { weizen }\end{array}$ & $\begin{array}{l}\text { Winter- } \\
\text { weizen }\end{array}$ & $\begin{array}{l}\text { Winter- } \\
\text { gerste }\end{array}$ & $\begin{array}{l}\text { Winter- } \\
\text { raps }\end{array}$ & $\begin{array}{l}\text { Winter- } \\
\text { raps }\end{array}$ & Erbse \\
$\begin{array}{l}\text { Fruchtfolgeanteil } \\
(\%)\end{array}$ & $33 \%$ & $15 \%$ & $18 \%$ & $19 \%$ & $14 \%$ & $2 \%$ \\
\hline
\end{tabular}

Das in diesem Beitrag verwendete Modell wurde in Anlehnung an die „Treibhausgasberechnungen Silomais“ des Kuratoriums für Technik und Bauwesen in der Landwirtschaft e. V. (KTBL) und der Landwirtschaftskammer Niedersachsen aufgebaut. Die Herleitung der vom KTBL erhobenen und im Modell angewandten Zahlen werden im „Handbuch - Berechnungsstandard für einzelbetriebliche Klimabilanzen (BEK) in der Landwirtschaft" aufgeführt. Diese sind auch Grundlage für die Analysen im Bereich der Tierhaltung (Kap. 4).

Das Berechnungsmodell ist auf den Zeitraum eines Wirtschaftsjahres begrenzt und bildet somit eine Anbauperiode ab. Der Rahmen der Betrachtung wird dabei ab Aussaat bis zur Ernte des Getreides bzw. Rapses gesteckt. Die Erzeugung des Saatgutes beispielsweise bzw. der Transport, die Lagerung und der Verkauf des Erntegutes werden nicht separat ausgewiesen. Der Betriebsmitteleinsatz wie Diesel, Dünger und Pflanzenschutz wird je Hektar auf die jeweilige Kultur angewandt.

Für jede Kultur fließen der Anbauumfang und der jeweilige Ertrag in die Berechnungen ein. Wesentliche Quellen für Emissionen von Treibhausgasen sind auf die Düngung zurückzuführen, deshalb ist bei der Analyse Detailschärfe erforderlich. Z. B. wird Mineraldünger unterteilt in Stickstoff (N) aus Harnstoff, Stickstoff aus Ammoniumnitrat-Harnstoff-Lösung (AHL) und Stickstoff aus weiteren Quellen. Außerdem finden Phosphor-, Kalium- und Kalkdüngung Eingang in die Berechnungen.

Die Anbaufrüchte und ihr Ertragspotential werden je nach Vorkultur unterschieden, da die Vorfruchtwerte ebenfalls berücksichtigt werden. Da das Stroh auf dem Acker verbleibt, wird dieses als Nährstofflieferung für die Werte $\mathrm{N}, \mathrm{P}_{2} \mathrm{O}_{5}$ und $\mathrm{K}_{2} \mathrm{O}$ angerechnet.

Des Weiteren werden Diesel- und Biodieselverbrauch pro Hektar, Saatguteinsatz in Körnern je Hektar und Einsatz von Pflanzenschutzmitteln gemittelt in Liter pro Hektar für jede Anbaufrucht ausgewiesen.

Als Ergebnis und Vergleichswert wird der Mittelwert über die verschiedenen Kulturen gebildet und stellt so den THG-Jahreswert in $\mathrm{kg} \mathrm{CO}_{2 \text { eq }}$ pro Hektar dar.

\section{Diskutierte Möglichkeiten der $\mathrm{CO}_{2}$-Reduzierung durch}

- Diesel ersetzen z. B. durch E-Mobilität oder die Brennstoffzelle (Szenario I)

- Fruchtfolgeumstellungen, Steigerung des Leguminosenanteils in der Fruchtfolge (Szenario II)

- Extensivierung des Anbaues durch schrittweise Reduzierung der N-Düngung, abnehmende Erträge um $10 \mathrm{dt} / \mathrm{ha}$ bzw. $20 \mathrm{dt} / \mathrm{ha}$ (Szenario III-IV) 
Tab. 2 Simulationsergebnisse für einen Ackerbaubetrieb in Mecklenburg-Vorpommern. (Quelle: Betriebsdaten, Simulation in Anlehnung an die „Treibhausgasberechnungen Silomais“ des Kuratoriums für Technik und Bauwesen in der Landwirtschaft e. V. (KTBL))

\begin{tabular}{|c|c|c|c|c|c|}
\hline \multirow[t]{2}{*}{ Szenarien } & \multirow[t]{2}{*}{$\begin{array}{l}\text { Status } \\
\text { quo } \\
(2019)\end{array}$} & \multirow{2}{*}{$\begin{array}{l}\text { Diesel ersetzen, z. B. } \\
\text { E-Mobilität oder } \\
\text { Brennstoffzelle }^{1)} \\
\text { I }\end{array}$} & \multirow{2}{*}{$\begin{array}{l}\text { Fruchtfolge } \\
\text { ändern, mehr } \\
\text { Leguminosen } \\
\text { II }\end{array}$} & \multicolumn{2}{|c|}{$\begin{array}{l}\text { Extensivierung, weniger } \\
\text { N-Dünger } \\
(\mathrm{III}-10 \mathrm{dt}, \mathrm{IV}-20 \mathrm{dt})\end{array}$} \\
\hline & & & & III & IV \\
\hline $\begin{array}{l}\text { Summe THG, } \\
\mathrm{kg} \mathrm{CO}_{2} \mathrm{eq} / \mathrm{ha}\end{array}$ & 1930 & 1541 & 1853 & 2115 & 2301 \\
\hline $\begin{array}{l}\mathrm{CO}_{2} \text {-Fußabdruck, } \\
\mathrm{kg} \mathrm{CO}_{2} \mathrm{eq} / \mathrm{kg} \mathrm{TM}\end{array}$ & 0,352 & - & 0,365 & 0,468 & 0,669 \\
\hline 1) ohne Diesel & - & 0,279 & 0,286 & 0,381 & 0,558 \\
\hline
\end{tabular}

Die Ergebnisse der Szenarien I (Diesel ersetzen), II (Fruchtfolge ändern), III (Extensivierung, - $10 \mathrm{dt}$ Ertrag) und IV (Extensivierung, - 20 dt Ertrag) werden folgend kurz zusammengefasst in hinsichtlich ihrer $\mathrm{CO}_{2}$-Minderungseffekte eingeschätzt

Durch den Einsatz von Leguminosen kann der $\mathrm{CO}_{2}$-Fußabdruck reduziert werden. Eine zusätzliche Extensivierung, d.h. Reduzierung der N-Düngung lässt die $\mathrm{CO}_{2 \mathrm{eq}}$-Emission jedoch wieder ansteigen; hier wäre sogar eine Verschlechterung gegenüber der Ausgangssituation festzustellen (Tab. 2). Der größte Effekt wird erzielt, wenn die vorgelagerte Industrie bereits $\mathrm{CO}_{2}$-neutral die eingesetzten Betriebsmittel (Maschinen, Saatgut, Dünger, Pflanzenschutzmittel) liefern könnte und der landwirtschaftliche Betrieb seinen eigenen Energiebedarf, hier hauptsächlich Diesel, durch eigenerzeugte Erneuerbare Energien decken würde.

\subsection{Schlussfolgerungen zu den Szenarien I bis IV}

- In den betrachteten Fällen (I bis IV) führt der Ersatz der fossilen Energieträger $\mathrm{zu}$ einem verbesserten $\mathrm{CO}_{2}$-Fußabdruck. Wird jedoch unterstellt, dass diese durch erneuerbare Energien kompensiert werden müssten, stellt sich speziell beim Maschinenantrieb die Frage, welche zusätzlichen Kosten dadurch entstehen würden.

- Die Umstellung der Fruchtfolge zu einem höheren Leguminosen-Anteil verzeichnet eine geringe Wirksamkeit. In Szenario II wurde in der Fruchtfolge der Anteil der Leguminosen von 2\% auf $17 \%$ erhöht. Der Anbau von Winterweizen nach Winterweizen wurde im Gegenzug komplett gestrichen. Die geringeren Erträge der Kultur Erbse im Vergleich zum Winterweizen führen zu einem ähnlichen THG-Wert. Dennoch ist die Erbse als stickstoffliefernde Frucht in Hinblick auf die klimagasrelevante Bedeutung deutlich positiver zu bewerten als das Wintergetreide, was nicht zuletzt auch am positiven Vorfruchtwert der Erbse liegt.

- Die reduzierte Intensität der Stickstoffdüngung in Szenario III ist als wenig vorteilhaft zu bewerten. Durch die Reduzierung der Stickstoffdüngung verschiebt sich das Input-Output-Verhältnis, sodass es in Folge zu einer relativen Erhöhung der THG-Emissionen pro Output kommt.

- Wird die Stickstoffdüngung reduziert, ist zu erkennen, dass die THG-Emissionen steigen. Die Senkung der N-Düngung nach Entzug und die entsprechende Annahme, dass sich damit auch der Ertrag um 10 bzw. 20 dt verringert, führen in diesem 
Fall zu steigenden Klimabelastungen. Die eingesparten THG aus verringertem Betriebsmitteleinsatz sind somit nicht ausreichend, um den geringeren Ertrag und die damit einhergehenden THG-Minderungseffekte aufzufangen. Dies deutet darauf hin, dass die singuläre Reduzierung der Stickstoffdüngung am Beispiel des Düngemittel Harnstoff als einzelne Lösungsoption kritisch zu hinterfragen ist.

Das zur Berechnung des $\mathrm{CO}_{2}$-Fußabdruckes im Ackerbau heran gezogene Modell unterscheidet bei den einzelnen Produkten nicht im Hinblick auf verschiedene Arten der Bodenbearbeitung. Wendende und nichtwendende Bodenbearbeitung setzen unterschiedliche THG-Emissionen pro ha voraus Feike et al. (2020). Des Weiteren bildet das Modell das Beispieljahr 2019 ab. Es ist zu erwarten, dass die THGEmissionen und der $\mathrm{CO}_{2}$-Fußabdruck der einzelnen Kulturen sich wetterbedingt und durch die unterschiedliche Einsatzmenge von Dünger und Pflanzenschutzmitteln verändern könnten. Unterstützend dazu zeigen sich bei den Versuchen von Feike et al. (2020), dass ein Verzicht auf Fungizide zu einem deutlichen Ertragsrückgang führt, der wiederum den $\mathrm{CO}_{2}$-Fußabdruck des nicht mit Fungiziden behandelten Getreides verschlechtert.

Damit deutet sich an, das einfache Lösungsansätze, wie die singuläre Variation von Inputmengen (weniger Dünger, mehr Leguminosen u. a.) nicht automatisch zu einer nachhaltigen Verbesserung der $\mathrm{CO}_{2}$-Bilanz eines Betriebes führen müssen. Der zielgerichtete Einsatz digitaler Technologien könnte diesbezüglich wertvolle zusätzliche Lösungsmöglichkeiten bieten.

\subsection{Ackerbau - wie kann die Digitalisierung helfen die Klimabilanz zu verbessern?}

Die Akzeptanz und der Einsatz neuer Technologien ist immer auch im Lichte der wirtschaftlichen Effekte für die Anwender zu bewerten. Im Folgenden werden verschiedene Anwendungsbereiche für Precision Farming Anwendungen mit Blick auf ihre möglichen Einsparpotenziale beispielhaft dargestellt. Dazu zählen Anwendungen der Fernerkundung, wie Satelliten-Biomassekarten, digitale Geländemodelle, Bodenleitfähigkeitsmessungen und Luftbilder. Darüber hinaus werden digitale Ertragskarten sowie Bodenbonitätskarten, hier bezeichnet als Applikationskarten betrachtet. Der Einsatz von Stickstoff-Sensoren zur Ermittlung des aktuellen Stickstoffbedarfes wird zusammengefasst unter Informationen aus Sensordaten. Der Einsatz von Satellitenortungssystemen zur präzisen und überlappungsfreien Steuerung von Maschinen auf dem Feld wird unter der Rubrik automatische Lenksysteme eingeordnet. Die zugrunde gelegten Daten wurden im Rahmen einer Masterabschlussarbeit in den Fachgebieten Agrarökonomie und Landtechnik im Jahr 2016 ermittelt. Im Rahmen der Arbeit wurden Anbausysteme zur teilflächenspezifischen Bewirtschaftung für die Bereiche Düngung, Saatgutausbringung und Pflanzenschutz für den Ackerbau eines norddeutschen Landwirtschaftsbetriebes untersucht. Für die folgende Darstellung wurde eine klassische Winterraps-Winterweizen-Wintergersten-Fruchtfolge unterstellt (Tab. 3).

Die Summe der möglichen Einsparpotenziale liegt für die Kulturen Winterraps, Winterweizen und Wintergerste zwischen 65 und $71 €$ pro ha und beruht im We- 
Tab. 3 Precision Farming Anwendungen und mögliche Einsparpotenziale in EUR/ha. (Quelle: Bieber 2016, verändert)

\begin{tabular}{|c|c|c|c|c|}
\hline \multirow{2}{*}{$\begin{array}{l}\text { Precision Farming } \\
\text { Anwendungen }\end{array}$} & \multirow[t]{2}{*}{ Anwendungsbereich } & \multicolumn{3}{|c|}{ Einsparpotenziale (EUR/ha) } \\
\hline & & $\begin{array}{l}\text { Winterraps } \\
(€)\end{array}$ & $\begin{array}{l}\text { Winterweizen } \\
(€)\end{array}$ & $\begin{array}{l}\text { Wintergerste } \\
(€)\end{array}$ \\
\hline \multirow{2}{*}{$\begin{array}{l}\text { Fernerkundung, } \\
\text { Applikationskarten }\end{array}$} & Wachstumsregler & - & 15 & 14 \\
\hline & $\begin{array}{l}\text { Teilflächenspezifische } \\
\text { Aussaat }\end{array}$ & - & 9 & 7 \\
\hline \multirow{3}{*}{$\begin{array}{l}\text { Applikationsdaten, } \\
\text { Sensordaten }\end{array}$} & N-Düngung & 24 & 13 & 11 \\
\hline & P-Düngung & 17 & 8 & 8 \\
\hline & K-Düngung & 5 & 3 & 3 \\
\hline $\begin{array}{l}\text { Automatische Lenk- } \\
\text { systeme }\end{array}$ & $\begin{array}{l}\text { Aussaat, Düngung, Pflan- } \\
\text { zenschutz }\end{array}$ & 25 & 22 & 21 \\
\hline \multicolumn{2}{|c|}{ Summe der Einsparpotenziale pro Kultur (EUR/ha) } & 71 & 70 & 65 \\
\hline
\end{tabular}

sentlichen auf Einsparungen im Bereich der Direktkosten (Aufwendungen für Dünger, Saatgut, Pflanzenschutz). Hinzukommen verminderte Aufwendungen aus dem Bereich der Arbeitserledigungskosten (Lohnkosten, Dieselverbrauch, Maschinenstunden) durch den Einsatz automatischer Lenksysteme. Neben den positiven ökonomischen Effekten durch die reduzierten Aufwandmengen, sind Einsparungen im Bereich der THG-Emissionen vorstellbar. Diese wurden in den hier dargestellten Überlegungen jedoch unberïcksichtigt gelassen.

Im Folgenden werden die bisher dargestellten Betrachtungen zu $\mathrm{CO}_{2}$-Minderungspotenzialen und Lösungsmöglichkeiten der Digitalisierung im Ackerbau um den Bereich der ökologischen und konventionellen Tierhaltung erweitert und am Beispiel eines Milchviehbetriebes in Mecklenburg-Vorpommern beschrieben.

\section{$4 \mathrm{CO}_{2}$-Fußabdruck in der Tierhaltung - am Beispiel der ökologischen Milchproduktion - Möglichkeiten der Reduzierung}

Die weltweiten THG-Emissionen von Rindern betragen $5335 \mathrm{Mt} \mathrm{CO}_{2}$-Äquivalente $\left(\mathrm{CO}_{2}\right.$ eq) pro Jahr, das entspricht $11 \%$ der von der Weltbevölkerung verursachten THG-Emissionen (Smith et al. 2014).

Die Milchhaltung trägt damit wesentlich zu den Gesamtemissionen auf der Erde bei. Mit $72 \%$ entsteht der größte Anteil der Emissionen, bevor die Milch den Landwirtschaftsbetrieb verlässt (Thoma et al. 2013). Dabei umfassen die wichtigsten Quellen für die THG-Emissionen den Prozess der enterischen Fermentation während der Verdauung im Wiederkäuermagen der Tiere, die Fütterung und Haltung der Milchkühe (I), die Lagerung und Verwendung von Gülle (II-III), sowie die Ernte des Ackerfutters (IV) und die Beweidung des Grünlandes (V) (Tab. 4).

Die aufgezeigten direkten und indirekten Treibhausgasquellen (I bis V) können betriebsindividuell sehr unterschiedlich wirken. Dabei wird die absolute Höhe der Emissionen eines Milchviehhaltenden Betriebes durch Faktoren wie beispielsweise die Art der Haltung (Anbindehaltung, Laufstallhaltung, Freilandhaltung), der Fütterung (im Stall oder durch Weide) oder durch die Art der Bodenbeschaffenheit im 
Tab. 4 Direkte und indirekte THG-Quellen und ansteigende relative Mengen (wenig +, viel ++, sehr viel $+++)$ aus Milchviehbetrieben. (Quelle: Rotz 2018, verändert)

\begin{tabular}{|c|c|c|c|c|c|c|}
\hline $\mathrm{Nr}$ & Direkte/indirekte THG-Quellen & $\mathrm{CO}_{2}$ & $\mathrm{CH}_{4}$ & $\mathrm{~N}_{2} \mathrm{O}$ & $\mathrm{NH}_{3}$ & $\mathrm{NO}_{3}$ \\
\hline \multirow[t]{5}{*}{ I } & Milchkühe (enterische Fermentation) & & +++ & + & & \\
\hline & Fütterung & + & & & & \\
\hline & Anbindehaltung & & + & & ++ & \\
\hline & Laufstallhaltung & & + & & ++ & \\
\hline & Einstreu der Liegeflächen & & ++ & + & +++ & \\
\hline \multirow[t]{3}{*}{ II } & Güllelagerung, separierte Feststoffe & & + & ++ & +++ & \\
\hline & Güllelagerung, flüssig/fest & & ++ & ++ & +++ & \\
\hline & Güllelagerung, flüssig & & ++ & + & +++ & \\
\hline III & Gülleausbringung, Injektion & + & & & + & \\
\hline IV & Ackerland & & & ++ & + & ++ \\
\hline $\mathrm{V}$ & Weideland & & & ++ & ++ & ++ \\
\hline
\end{tabular}

Laufbereich der Tiere (Betonboden oder Stroheinstreu) mit beeinflusst. Damit wirken neben den produktionstechnischen Zusammenhängen für die Bildung von THGEmissionen auch Managemententscheidungen, wie die Entscheidung für eine ökologische oder konventionelle Wirtschaftsweise, auf die Klimabilanz eines Betriebes ein.

Aufgrund dieser unterschiedlich stark wirkenden Einflussfaktoren wird die nachfolgende Modellrechnung zur Bestimmung des $\mathrm{CO}_{2}$-Fußabdrucks exemplarisch für einen ökologisch wirtschaftenden Milchviehbetrieb mit Sitz in Mecklenburg-Vorpommern erläutert. Zur Berechnung wird zusätzlich ein konventioneller Milchviehbetrieb auf Basis von Praxiszahlen gegenübergestellt.

Der ökologische Betrieb bewirtschaftet 120 ha Acker sowie 300 ha Dauergrünland. Der Pflanzenbau wird ausschließlich zur Futterproduktion für die Rinder betrieben. Im Tierbestand befinden sich 130 Milchkühe und ihre Nachzucht, zusammen weitere 120 Zuchtkälber und -färsen. Die männlichen Kälber der Milchrinder werden im Alter von sechs Wochen zur Mast weiterverkauft. Der gesamte Betrieb wird nach den EU-Richtlinien des Ökologischen Landbaus bewirtschaftet. Somit sind in den Berechnungen keine Pflanzenschutzmittel zum Einsatz gekommen und keine mineralischen Dünger. Ein Grundgedanke des Ökolandbaus ist die Kreislaufwirtschaft, d.h. die Ausscheidungen der Tiere dienen wiederum als Nährstoffe für die Pflanzen. In den Klimagasberechnungen zum Pflanzenbau wurde eine Nährstoffversorgung mit Gülle unterstellt. Eine weitere Besonderheit besteht in der Fütterung der Rinder. Im Vergleich zu konventionellen Milchviehbetrieben wird im Ökolandbau häufig Kleegras in Form von Silage oder Heu verwendet. Zur Berechnung des $\mathrm{CO}_{2^{-}}$ Fußabdruckes in der Milchproduktion wurde für die Szenarien I und II eine durchschnittliche Milchleistung von 7500 bzw. $9200 \mathrm{~kg}$ Milch pro Tier und Jahr unterstellt. Die Futterration der Vergleichsszenarien unterscheidet sich aufgrund des restriktiven Einsatzes von Kraftfutter in der ökologischen Milchviehhaltung. In der Variante der ökologischen Milcherzeugung (Szenario I) wurde ausschließlich Kleegrassilage und minimal Kraftfutter verfüttert. Die Variante der konventionellen Milcherzeugung (Szenario II) beinhaltet die Futterkomponenten Maissilage, Grassilage und 
Tab. 5 THG-Emissionen für das Hauptprodukt (Milch ${ }^{\mathrm{a}}$ ) für einen ökologisch wirtschaftenden Milchviehbetrieb in M-V im Vergleich zur konventionellen Milcherzeugung. (Quelle: eigene Berechnungen)

\begin{tabular}{llll}
\hline & $\begin{array}{l}\text { Szenarien der Milcherzeu- } \\
\text { gung } \\
\text { Einheit }\end{array}$ & Ökologisch & Konventionell \\
\hline Milchleistung pro Kuh und Jahr & $\mathrm{Kg} \mathrm{Milch/Kuh}$ & $\mathrm{I}$ & $\mathrm{II}$ \\
$\mathrm{CO}_{2}$-Fußabdruck pro Kuh und & $\mathrm{Kg} \mathrm{CO} 2 \mathrm{eq} / \mathrm{Kuh}$ & 7500 & 9200 \\
$\mathrm{Jahr}$ & & 4255 & 7576 \\
$\mathrm{CO} 2-\mathrm{Fußabdruck}$ pro kg Milch & $\mathrm{Kg} \mathrm{CO} 2 \mathrm{eq} / \mathrm{kg}$ Milch & 0,57 & 0,85 \\
\hline
\end{tabular}

aAlle Mengenangaben sind normiert auf den Standard „Milch mit 4,0\% Fett und 3,4\% Eiweiß“ (Energiekorrigierte Milch (ECM))

Kraftfutter inklusive Sojaschrot. Für beide Szenarien wurde die Haltungsform eines Liegeboxenlaufstalls mit einem zeitlich begrenzten Weidegang von Frühjahr bis Herbst unterstellt. Folgend ist die $\mathrm{CO}_{2}$ Bilanz für einen Milchviehbetrieb in ökologischer Wirtschaftsweise (Szenario I) und konventioneller Wirtschaftsweise (Szenario II) gegenübergestellt (Tab. 5).

Der $\mathrm{CO}_{2}$-Fußabdruck im hier dargestellten Vergleich ist in der ökologischen Milchviehhaltung (Szenario I) um etwa ein Drittel geringer als bei der konventionellen Milchproduktion (Szenario II). Die vergleichsweise bessere $\mathrm{CO}_{2}$-Bilanz der ökologischen Variante liegt insbesondere in den positiven Effekten $\left(\mathrm{CO}_{2}\right.$-Minderungseffekten) des Humusanreicherungspotentials und an der Luft-Stickstoffbindung der Kleepflanzen begründet. Die im Vergleich dazu schlechtere $\mathrm{CO}_{2}$-Bilanz im Szenario II ist auf den Einsatz von Mais-Grassilage und Kraftfutter zurückzuführen.

Im angestellten Vergleich fällt der $\mathrm{CO}_{2}$-Fußabdruck der ökologischen Variante um $0,28 \mathrm{~kg} \mathrm{CO} 2 \mathrm{eq} / \mathrm{kg}$ Milch niedriger aus als in der konventionellen Variante mit $0,85 \mathrm{~kg}$ $\mathrm{CO}_{2} \mathrm{eq} / \mathrm{kg}$ Milch. Die Ergebnisse deuten auf Unterschiede in den $\mathrm{CO}_{2}$-Bilanzen der beiden Produktionsverfahren hin. Insgesamt ist davon auszugehen, das $\mathrm{CO}_{2}$-Bilanzen aufgrund der Vielzahl von Einflussfaktoren (Milchleistung, Fütterungsintensität, Betriebsorganisation, technische Ausstattung u.a.) innerhalb und zwischen einzelnen Produktionssystemen (ökologisch, konventionell) stark variieren. Die jeweiligen betrieblichen Zusammenhänge beeinflussen die absoluten Emissionen damit nicht unwesentlich.

\subsection{Milchproduktion - wie kann die Digitalisierung helfen die Klimabilanz zu verbessern?}

Precision Livestock Farming Technologien wie Melkroboter, Sensoren zur Aktivitätsmessung der Tiere oder Farmmanagementprogramme ermöglichen eine hohe technische Effizienz in der Milchproduktion. Dabei dient die technische Effizienz insbesondere dazu die Kosten und Leistung des Produktionszweiges Milch transparenter und damit steuerbarer zu machen. Die Messung von Umwelteffekten (z.B. $\mathrm{CO}_{2}$-Output pro Liter Milch) steht dabei überwiegend nicht im Fokus. Dabei könnten digitale Anwendungen durch die Steigerung der betriebsindividuellen technischen Effizienz implizit dazu beitragen, die Umwelteffizienz positiv zu beeinflussen. Insgesamt ist davon auszugehen, dass technisch effiziente Produktionssysteme relativ zum 
Output weniger Emissionen erzeugen als weniger effiziente Systeme. Im folgenden Abschnitt werden exemplarisch Ansatzstellen (I. Tiergesundheit, II. Melkprozess, III. Energieverbrauch im Stall) zur Steigerung der technischen Effizienz aufgeführt.

\section{Tiergesundheit}

- Responder ermitteln Daten zu Fresszeiten, Körpertemperatur und Aktivität eines Tieres. Die Auswertung und Analyse von Aktivitätsraten oder Fressverhalten der Tiere ermöglichen eine tierindividuelle Entscheidungsfindung unabhängig von der Tieranzahl des Betriebes.

- Pedometer und Sensoren im Laufbereich zum Melkstand erlauben Rückschlüsse über die Klauengesundheit der Tiere.

- Sensoren im Melkstand ermitteln die tierindividuelle Milchmenge, Fett- und Eiweißgehalt der gemolkenen Milch, Zellgehalt und Milchtemperatur. Dadurch sind Rückschlüsse auf die Tiergesundheit möglich.

\section{Melkprozess}

- In Kombination mit vollautomatischen Melksystemen ist eine automatische Kraftfutterzuteilung möglich. Die Zuteilung erfolgt dabei kuhindividuell, je nach Milchleistung und Laktationsstadium des Tieres. Die individuelle Versorgung der Tiere vermindert eine Über- bzw. Unterversorgung.

\section{Energieverbrauch im Stall}

- Der Energieverbrauch ist durch Wärmerückgewinnung aus gemolkener Milch und der Frequenzsteuerung von Geräten möglich. Elektrische Geräte wie automatische Futterschieber, Reinigungsroboter im Laufbereich der Tiere und Fütterungsroboter reduzieren den Kraftstoffverbrauch. Der zusätzliche Strombedarf kann durch erneuerbare Energien in Form einer Biogasanlage (Reststoffe wie Gülle), Photovoltaik (auf dem Stalldach) oder durch Windenergieanlagen ausgeglichen werden.

Die hier exemplarisch aufgeführten Ansatzstellen zu Tiergesundheit und Melkprozess (I-II) ermöglichen eine gezielte Analyse und Auswertung von Daten auf der Ebene von Einzeltieren. Die daraus ableitbaren Entscheidungen z. B. eine gezielte Klauenbehandlung oder zusätzliche Euterhygiene könnten die Gesundheit und damit die Nutzungsdauer der Tiere positiv beeinflussen. In einer Untersuchung von Brade und Wimmers (2016) wird darauf verwiesen, dass sich die Verbesserung der Tiergesundheit positiv auf den Methan-Output im Verhältnis zur Milchmenge auswirken könnte. Auf der Seite des betrieblichen Energiebedarfs (III) können die bereits genannten Verfahren zur Nutzung erneuerbarer Energien dazu beitragen die Klimabilanz der Milchproduktion positiv zu beeinflussen.

Neben den produktionstechnischen Stellschrauben zur Verbesserung der Klimabilanz in den Bereichen Ackerbau und Milchproduktion gibt es weitere Faktoren, die auf der Ebene von Entscheidungen wirken und im Folgenden Abschnitt analysiert werden. 


\section{Umsetzungsvoraussetzungen und Erfolgsbedingungen in einer marktwirtschaftlich-unternehmerisch geprägten Landwirtschaft}

Die oben dargestellten Maßnahmen zur Reduktion der Klimawirkung durch die Digitalisierung über die Breite landwirtschaftlicher Produktionsverfahren hinweg stellen zunächst modellhafte Optionen dar, die zur Entfaltung ihrer Wirkungen den Weg in die landwirtschaftliche Praxis finden müssen. Angesichts der privatwirtschaftlichen Verfasstheit der landwirtschaftlichen Unternehmungen, der Strukturvielfalt der insgesamt ca. 260.000 landwirtschaftlichen Betriebe in Deutschland (Statistisches Bundesamt 2019) und der europäisch angelegten gemeinsamen Agrarpolitik bedarf die zügige Einführung von Digitallösungen im Interesse des Klimaschutzes eines erweiterten Blicks auf einige Besonderheiten der Branche.

Landwirtschaft ist als Experimentierfeld insbesondere im Zusammenhang mit Precision oder Smart Farming als Weg zur Effizienzsteigerung unter Verringerung von Betriebsmitteln und/oder der Erhöhung der Erträge durch teilflächenspezifische Maßnahmen und Applikationen grundsätzlich sehr geeignet für die Implementierung technischer Fortschritte in der Automatisierung beispielsweise von Lenksystemen. Anders als beim autonomen Fahren im Straßenverkehr sind landwirtschaftliche Fahrzeuge in der ,geschützten Atmosphäre“ abgegrenzter Flächen unterwegs. Viele Risiken des autonomen Fahrens können hier erprobt oder ausgeschlossen werden. Das liegt u. a. daran, dass landwirtschaftliche Arbeitsmaschinen sich weit überwiegend nur zu ausgewählten Zeitpunkten (Aussaatvorbereitung, Aussaat, Bestandspflege, Ernte) und auf Flächen mit wohldefinierten Eigentumsrechten bewegen. Diese Vorteile gehen jedoch mit der Voraussetzung einher, bei der Einführung klimaschonender Digitallösungen üblicherweise von der Zustimmung bzw. der aktiven Entscheidung zugunsten eines entsprechenden Verfahrens durch die Unternehmensleitung bzw. die landwirtschaftliche Betriebsleitung abhängig sind. Die aber kann nicht als selbstverständlich gegeben angenommen werden, insbesondere dann, wenn der Einsatz klimaschonender Produktionsverfahren mit erhöhtem Investitions- oder Betriebsmitteleinsatz verbunden ist.

Eine der Allgemeinheit zugutekommende Maßnahme dürfte nur dann in Erwägung gezogen werden, wenn nicht a priori davon auszugehen ist, dass die mit ihr verbundenen Aufwendungen privatisiert werden. Alternative, nichtmonetäre Umsetzungsmechanismen, die etwa auf Ge- und Verbote setzen würden, ließen erhebliche Gegen- und Ausweichreaktionen erwarten. Aktuelle Beispiele wären Demonstrationsmaßnahmen etwa im Zusammenhang mit Dünge- oder Insektenschutzverordnungen.

Eine Grundanforderung an den breiten Einsatz digitaler Technologien auch zugunsten des Klimaschutzes besteht in einer ausreichenden Netzabdeckung bzw. Netzverfügbarkeit in erforderliche Übertragungsgeschwindigkeit und -qualität. Hier gibt es erhebliche Versorgungslücken im ländlichen Raum (Bundesnetzagentur 2019).

Die Anforderungen an Investitionen in veränderte Produktionsverfahren werden zusätzlich dadurch erhöht, dass insbesondere in den Familienbetriebsstrukturen zum Teil sehr langfristige Investitionszyklen zu beobachten sind. Daraus folgen entsprechend lange Innovationszyklen. Ein Traktor mit einem Investitionszeitraum von 
10 Jahren setzt damit einen technischen Standard für einen - insbesondere in der schnelllebigen Digitalisierungsumwelt - relativ langen Zeitraum. Ohne schlüssige Nachrüst-Optionen, die es ermöglichen auch ältere Maschinen in hohem Maße an kurzfristige technische Weiterentwicklungen oder sogar disruptiven Innovationssprüngen anzupassen, dürfte die Implementierung aktueller Klimaschutztechnologie deutlich erschweren.

Die Möglichkeit herstellerunabhängig unterschiedliche digitale Lösungen in der Vielfalt der Landtechnik miteinander zu kombinieren ist bis zum heutigen Zeitpunkt eingeschränkt. Eine faktische Festlegung auf eine digitale Technologie verlangt Investitionssicherheit dahingehend, dass eine Investitionsentscheidung im Vertrauen auf die Kompatibilität mit Anbaugeräten oder anderen Maschinen in der Peripherie der Investition gefällt werden kann. Dieser Punkt ist auch vor dem Hintergrund von Bedeutung, dass die Digitalisierung der Landtechnik seit Jahren Einzug in die landwirtschaftlichen Betriebe hält. Nicht selten folgte diese Form der schleichenden oder immerhin schrittweisen Digitalisierung keinem Masterplan, der zu geschlossenen ITSystemen in den Unternehmen und auf den Betrieben geführt hätte. Dieses - nicht auf die Landwirtschaft beschränkte Phänomen - kann zu den Grundherausforderungen der Digitalisierung gezählt werden. Beispiele aus der Welt des Automobilbaus, des Bankings oder des stationären Handels weisen vergleichbare Muster auf - und begünstigen offenkundig disruptive Lösungen.

Datensicherheit und Datenverfügbarkeit spielen eine herausragende Rolle dabei, Vertrauen in die Nutzung von Digitallösungen zu unterstützen (DLG 2018). Als Anforderung wird die Erwartung formuliert, dass die Daten im Eigentum des Landwirts bzw. des landwirtschaftlichen Betriebs verbleiben. Deren Nutzung müsste auf klar definierte Zwecke limitiert sein und nachweislich gewährleistet werden. Vor dem Hintergrund des hohen Verflechtungsgrades der Landwirtschaft mit ihren vorund nachgelagerten Bereichen stellt sich die Frage nach Schnittstellen-Definitionen, mit denen digitale Systeme unterschiedlicher Akteure (Landwirt - Landtechnik Landhandel) so miteinander kommunizieren können, dass operative Vorteile erzielbar werden, ohne dass die Risiken, die aus dem „Five-Forces-Modell“ (Porter 1979) für das „Geschäftsmodell Landwirtschaft" folgen, überhandnehmen. Bei einer ungleichgewichtigen Vernetzungsstrategie könnten die Macht der Zulieferer oder die Macht der Kunden zuungunsten der Landwirtschaft relativ anwachsen.

Mit dem Grad der Digitalisierung steigen nicht nur Präzision und Produktivität, sondern häufig auch der Bedarf an Beratung und Qualifizierung. Investitionsentscheidungen werden komplexer, eine unabhängige Beratung zur Entwicklung einer Digitalinvestitionsstrategie erfordert Sachverstand, der nicht nur den einzelnen landwirtschaftlichen Anwendungsbereich, sondern auch die Breite der digitalen Lösungsmöglichkeiten im Blick hat. Die Nutzung aller Möglichkeiten, die in komplexen Lösungen verfügbar gemacht werden können, setzt häufig Fachkenntnisse auf der Anwenderebene voraus, die nicht zwangsläufig vorausgesetzt werden können. Hier sind Qualifizierungsmaßnahmen erforderlich, um den Umfang der Nutzwirkungen einer Investition in digitale Technik aktivieren zu können.

Die Verbreitung der zur deutlichen Verbesserung der Klimawirkungen der Landwirtschaft erforderlichen und in Teilen durchaus auch verfügbaren Technologien ist nicht nur von der Brillanz der technischen Lösung abhängig, sondern auch von deren 
Akzeptanz bei den Entscheidungsträgern und der Anwendungskompetenz der Nutzer in der Praxis. Diese Anforderungen sind nicht unmöglich zu erfüllen, sie sind aber nach aller Erfahrung auch nicht als selbstverständlich gegeben anzunehmen. Erforderlich ist also ein kluges, vertrauensvolles Zusammenwirken von Technik-Anbietern und Technik-Nutzern mit transparenten Erwartungshorizonten und Erfolgsbeiträgen für alle Seiten. Eine Unterstützung dieses Zusammenwirkens durch die (Agrar-)Politik ist denkbar und könnte bereits im Zusammenhang mit dem Greendeal-Konzept der EU-Kommission sowie der kommenden Finanzrahmenplanung und der damit im Zusammenhang stehenden Finanzierung der Agrarpolitik zur Geltung kommen. Als weiteres Forschungsfeld mit Handlungsbedarf erweist sich die wirtschafts- und sozialwissenschaftlich fundierte Identifikation und Interpretation der Attitüde der landwirtschaftlichen Entscheidungsträger gegenüber Digitalisierungstendenzen in der Landwirtschaft.

\section{Fazit}

Die Klimawirkungen der Landwirtschaft insgesamt sind vielfältiger Natur und die Einflussfaktoren auf die jeweilige Klimabilanz eines Betriebes so individuell wie die Betriebe selbst. Neben der Wirtschaftsweise (konventionell oder ökologisch) beeinflussen der Grad der betriebsindividuellen Technisierung (Handarbeit, Traktor, Roboter), die Art der genutzten Verfahren (Grubber oder Pflug, Stallhaltung oder Weidehaltung) und die naturräumliche Ausstattung (Bodenart, Geländebeschaffenheit, Niederschlagsmenge, das Verhältnis von Ackerland zu Grünland) die $\mathrm{CO}_{2}$ Bilanz eines Betriebes. Passende Lösungsmöglichkeiten und Ansatzstellen bedürfen daher einer gründlichen und möglichst individuellen Prüfung der betrieblichen Gegebenheiten. Digitale Anwendungen könnten hier eine wichtige Hilfestellung bieten. Der digitale Datenstrom kann dazu beitragen noch vorhandene Effizienzlücken zu finden und angemessen schließen zu können.

Die Facettenhaftigkeit der einzelnen Lösungsansätze deutet an, dass es keine Patentrezepte für einzelne Betriebe geben kann und das die Wirksamkeit der einzelnen Maßnahmen immer auch im Lichte der Wirtschaftlichkeit betrachtet werden sollte. Die im Rahmen der Klimastrategie-2050 geforderte $\mathrm{CO}_{2}$-Neutralität Europas sollte sich auch daran messen lassen.

Die Klimarelevanz der Landwirtschaft positiv zu beeinflussen darf nicht nur als Notwendigkeit verstanden werden, von einem unbefriedigenden Zustand A in einen befriedigenden Zustand B zu wechseln, sie muss vielmehr als Prozess verstanden werden, der eindeutig erkennbar in Richtung des erwünschten Zustandes B weist. Das ist die Basis dafür diesen Prozess unterstützen und fördern zu können. Die Verfügbarkeit technischer und digitaler Lösungen spielt eine wichtige Rolle, nicht jedoch die einzige. Unternehmerische Expertise und Rentabilität haben gleichfalls wichtige, nicht zu unterschätzende Bedeutung.

Danksagung Die Autoren danken der Hochschule Neubrandenburg für die Unterstützung im Rahmen der hochschulinternen Forschungsförderung 2020.

Funding Open Access funding enabled and organized by Projekt DEAL. 
Open Access Dieser Artikel wird unter der Creative Commons Namensnennung 4.0 International Lizenz veröffentlicht, welche die Nutzung, Vervielfältigung, Bearbeitung, Verbreitung und Wiedergabe in jeglichem Medium und Format erlaubt, sofern Sie den/die ursprünglichen Autor(en) und die Quelle ordnungsgemäß nennen, einen Link zur Creative Commons Lizenz beifügen und angeben, ob Änderungen vorgenommen wurden.

Die in diesem Artikel enthaltenen Bilder und sonstiges Drittmaterial unterliegen ebenfalls der genannten Creative Commons Lizenz, sofern sich aus der Abbildungslegende nichts anderes ergibt. Sofern das betreffende Material nicht unter der genannten Creative Commons Lizenz steht und die betreffende Handlung nicht nach gesetzlichen Vorschriften erlaubt ist, ist für die oben aufgeführten Weiterverwendungen des Materials die Einwilligung des jeweiligen Rechteinhabers einzuholen.

Weitere Details zur Lizenz entnehmen Sie bitte der Lizenzinformation auf http://creativecommons.org/ licenses/by/4.0/deed.de.

\section{Literatur}

\section{Verwendete Literatur}

Bieber M (2016) Ausgewählte ökonomische Effekte von Satellitenortungssystemen im Ackerbau. Masterthesis im Studiengang Agrarwirtschaft, Hochschule Neubrandenburg

Bitkom Research (2020) Schon 8 von 10 Landwirten setzen auf digitale Technologien. https://www. bitkom.org/Presse/Presseinformation/Schon-8-von-10-Landwirten-setzen-auf-digitale-Technologien. Zugegriffen: 17. Okt. 2020

Brade W, Wimmers K (2016) Methan-Minderungspotenziale bei Wiederkäuern. Ber Landwirtsch. https:// doi.org/10.12767/buel.v94i1

Brankatschk G, Finkbeiner M (2017) Crop rotations and crop residues are relevant parameters for agricultural carbon footprints. Agron Sustain Dev 37:58. https://doi.org/10.1007/s13593-017-0464-4

Bundesministerium für Ernährung und Landwirtschaft (2019) Grundsätze für die Durchführung der guten fachlichen Praxis im Pflanzenschutz. https://www.bmel.de/DE/themen/landwirtschaft/pflanzenbau/ pflanzenschutz/gute-fachliche-praxis.html. Zugegriffen: 25. Nov. 2020

Bundesnetzagentur (2019) Breitbandmessung der Bundesnetzagentur. https://breitbandmessung.de/. Zugegriffen: 18. Okt. 2020

DLG (2018) Digitale Landwirtschaft - Ein Positionspapier der DLG. https://www.dlg.org/de/landwirtschaft/ themen/technik/digitalisierung-arbeitswirtschaft-und-prozesstechnik/digitale-landwirtschaft. Zugegriffen: 16. Okt. 2020

Europäische Kommission (2020) Vom Hof auf den Tisch - eine Strategie für ein faires, gesundes und umweltfreundliches Lebensmittelsystem. https://ec.europa.eu/info/sites/info/files/communicationannex-farm-fork-green-deal_de.pdf. Zugegriffen: 16. Okt. 2020 (Mitteilung der Kommission an das Europäische Parlament, den Rat, den Europäischen Wirtschafts- und Sozialausschuss und den Ausschuss der Regionen. Brüssel, den 20.05.2020. COM (2020) 381 final)

Feike T, Riedesel Freiherr zu Eisenbach L, Lieb R, Gabriel D, Sabboura D, Shawon AR, Wetzel M, Klocke B, Krengel-Horney S, Schwarz J (2020) Einfluss von Pflanzenschutzstrategie und Bodenbearbeitung auf den $\mathrm{CO}_{2}$-Fußabdruck von Weizen. J Kulturpflanz 72(7):311-326. https://doi.org/10.5073/ JfK.2020.07.08

Meeting of G20 Agriculture Ministers (2011) Action plan on food price volatility and agriculture, ministerial declaration, meeting of G20 agriculture ministers in Paris, 22 and 23 June 2011. http://agriculture. gouv.fr/G20-1-agriculture-en-premiere. Zugegriffen: 16. Okt. 2020

Myhre G, Shindell D, Breon FM, Collins W, Fuglestvedt J, Huang J, Koch D, Lamarque JF, Lee D, Mendoza B, Nakajima T, Robock A, Stephens G, Takemura T, Zhang H (2013) Anthropogenic and natural radiative forcing. In: Stocker TF, Qin D, Plattner GK, Tignor M, Allen SK, Boschung J, Nauels A, Xia Y, Bex V, Midgley PM (Hrsg) Climate change 2013: the physical science basis. Contribution of Working Group I to the Fifth Assessment Report of the Intergovernmental Panel on Climate Change. Cambridge University Press, Cambridge, New York

Porter ME (1979) How competitive forces shape strategy. In: Harvard business review—strategic planing 
Radermacher FJ (2015an) Zukunftsfähige Landwirtschaft, Globalisierung \& Welternährung - Sind wir noch zu retten? Vortrag anlässlich der Hochschultage für Ökosoziale Marktwirtschaft und Nachhaltigkeit am 10.12.2015 an der Martin-Luther-Universität Halle-Wittenberg

Rotz CA (2018) Modeling greenhouse gas emissions from dairy farms. J Dairy Sci 101(7):6675-6690. https://doi.org/10.3168/jds.2017-13272

Scherer B (2020) 40\% weniger Diesel-dank kühlem Kopf statt bleiernem Gasfuß. ATZ Heavy Duty 13(2):70. https://doi.org/10.1007/s35746-020-0081-z

Smith P, Bustamante M, Ahammad H, Clark H, Dong H, Elsiddig E, Haberl H, Harper R, House J, Jafari M, Masera O, Mbow C, Ravindranath N, Rice C, Robledo-Abad C, Romanovskaya A, Sperling F, Tubiello F (2014) Agriculture, forestry and other land use (AFOLU). In: Edenhofer O, Pichs-Madruga R, Sokona Y, Farahani E, Kadner S, Seyboth K, Adler A, Baum I, Brunner S, Eickemeier P, Kriemann B, Savolainen J, Schlömer S, von Stechow C, Zwickel T, Minx JC (Hrsg) Climate change: mitigation of climate change. Contribution of working group III to the fifth assessment report of the intergovernmental panel on climate change. Cambridge University Press, Cambridge, S 811-922

Statistisches Bundesamt (2019) Anzahl der Betriebe in der Landwirtschaft in Deutschland in den Jahren 1975 bis 2019. https://www.destatis.de/. Zugegriffen: 17. Okt. 2020

Thoma GJ, Popp JS, Nutter DW, Shonnard DR, Ulrich R, Matlock MD, Kim D, Neiderman Z, Kemper N, East C, Adom F (2013) Greenhouse gas emissions from milk production and consumption in the United States: a cradle-to-grave life cycle assessment circa 2008. Int Dairy J 31:S3-S14

Umweltbundesamt (2020) Beitrag der Landwirtschaft zu den Treibhausgas-Emissionen. https://www. umweltbundesamt.de/daten/landforstwirtschaft/beitrag-der-landwirtschaft-zu-den-treibhausgas. Zugegriffen: 14. Okt. 2020

United Nations (2015) Transformation unserer Welt: die Agenda 2030 für nachhaltige Entwicklung. UN, New York

Wettemann PJC (2017) Die Entwicklung der Produktivität von Marktfruchtbetrieben unter Berücksichtigung von THG-Emissionen. Ger J Agric Econ. https://doi.org/10.22004/ag.econ.303530

\section{Weiterführende Literatur}

Arbeitsgruppe BEK (2016) Berechnungsstandard für einzelbetriebliche Klimabilanzen (BEK) in der Landwirtschaft. www.ktbl.de. Zugegriffen: 5. Okt. 2020

EKoTech (2020) Effiziente Kraftstoffnutzung der Agrartechnik. https://www.ekotech-projekt.eu/. Zugegriffen: 14. Okt. 2020

Europäische Kommission (2020) A climate resillient Europe-prepare Europe for climate disruptions and accelerate the transformation to a climate resillient and just Europe by 2030. Report of the Mission Board for Adaptation to Climate Change, including Societal Transformation. https://doi.org/10.2777/ 69766

Löhr J (2019) Aufstand der Bauern - Protest in Berlin. https://www.faz.net/aktuell/politik/inland/protestin-berlin-warum-die-bauern-demonstrieren-16505279.html. Zugegriffen: 16. Okt. 2020 (aktualisiert am 26.11.2019)

Verband Deutscher Landwirtschaftlicher Untersuchungs- und Forschungsanstalten (2014) Standpunkt Humusbilanzierung - eine Methode zur Analyse und Bewertung der Humusversorgung von Ackerland. VDLUFA, Speyer 